\title{
Phonon density of states of nanocrystalline Fe prepared by high-energy ball milling
}

B. Fultz

California Institute of Technology, mail 138-78, Pasadena, California 91125

J. L. Robertson

Oak Ridge National Laboratory, P.O. Box 2008, Oak Ridge, Tennessee 37831

T. A. Stephens and L. J. Nagel

California Institute of Technology, mail 138-78, Pasadena, California 91125

S. Spooner

Oak Ridge National Laboratory, P.O. Box 2008, Oak Ridge, Tennessee 37831

(Received 28 December 1995; accepted for publication 19 February 1996)

We performed inelastic neutron scattering measurements on nanophase Fe powders prepared by high-energy ball milling. Neutron energy loss spectra were measured for two states of the material: (1) as milled, when the material had a characteristic nanocrystallite size of $12 \mathrm{~nm}$; and (2) annealed, when the material had a characteristic crystallite size of $28 \mathrm{~nm}$. The longitudinal peak in the phonon density of states (DOS) of the nanophase Fe was broadened, compared to that of the annealed material. We attribute this broadening to short phonon lifetimes in nanocrystals. The nanophase material also showed an enhanced density of states at low energies below $15 \mathrm{meV}$, which may indicate the presence of intercrystallite vibrations. These differences in phonon DOS should have only a small effect on the difference in vibrational entropy of nanocrystalline and larger-grained Fe.

(C) 1996 American Institute of Physics. [S0021-8979(96)00611-1]

\section{INTRODUCTION}

There has been strong recent interest in nanocrystalline materials, generally defined as materials with microstructural features of $100 \mathrm{~nm}$ or less. The interesting microstructureproperties relationships of nanostructured materials are often classified as "confinement effects" and "interface effects." An example of a confinement effect is the shift in frequency of optical absorption that occurs in semiconductors when the crystallite size is comparable to the size of the electron-hole exciton. ${ }^{1}$ An example of an interface effect is the surprisingly high ductility of nanocrystalline ceramic $\mathrm{TiO}_{2}$, which has been attributed to atom movements within its copious grain boundaries. ${ }^{2}$ A high density of grain boundaries is certainly a distinguishing feature of nanocrystalline materials, but there were also reports that nanocrystalline grainboundary structures are more disordered than grain boundaries in large-grained materials. ${ }^{3}$ Although some of these early claims of disorder have been shown to be overstated, ${ }^{4,5}$ in the present work we show evidence of nanocrystalline vibrations that involve the motion of stiff crystallites separated by weak intercrystallite forces consistent with disordered grain boundaries.

There were early reports that the heat capacity of nanocrystalline materials is significantly larger than that of large-grained materials. ${ }^{6}$ Unfortunately, contamination by interstitial helium may have vitiated these early observations. ${ }^{7}$ Some measurements of Debye-Waller factors have indicated that atoms in nanocrystals have larger thermal mean-square displacements than in large-grained materials. ${ }^{8-11}$ More recently, Suzuki and Sumiyama measured neutron inelastic scattering spectra on nanocrystalline Ta prepared by mechanical attrition. ${ }^{12}$ These authors reported a smoothing of features in the phonon density of states (DOS) of the nanocrystalline Ta (10 nm crystallite size), but no general softening of the vibrational spectrum. On the other hand, in a recent inelastic neutron-scattering study on nanocrystalline $\mathrm{Ni}_{3} \mathrm{Al}$ with a $7 \mathrm{~nm}$ crystallite size, ${ }^{13}$ a large enhancement of the phonon DOS at low energies was reported, although it was suggested that this could have been caused by quasielastic scattering by hydrogen in the material. The present work was undertaken to measure the phonon DOS in nanocrystalline $\mathrm{Fe}$, which is not expected to absorb a significant amount of hydrogen. For nanocrystalline $\mathrm{Fe}$ we report some enhancement of the phonon DOS at low energies, together with a smoothing of the longitudinal peak.

\section{EXPERIMENT}

Iron powders were made by mechanical attrition of pieces of Fe of $99.995 \%$ purity. The material was milled for $12 \mathrm{~h}$ in a Spex 8000 mixer/mill with hardened steel vials and balls and a ball-to-powder weight ratio of 5:1. For comparison, a second sample was prepared from some of this same powder by annealing at $500{ }^{\circ} \mathrm{C}$ in an evacuated quartz ampoule for $0.5 \mathrm{~h}$, and then followed by cooling at the rate of $50{ }^{\circ} \mathrm{C} / \mathrm{h}$. X-ray diffractometry was performed with an Inel CPS-120 diffractometer using Co $K \alpha$ radiation. A few grams of the powders were heated to $400{ }^{\circ} \mathrm{C}$ and analyzed for evolved hydrogen, oxygen, and nitrogen by a HewlettPackard 5890 gas chromatograph equipped with a thermal conductivity detector. We also analyzed the gas evolution from samples of $\mathrm{Ni}_{3} \mathrm{Al}$ which were used in a previous study. ${ }^{13}$

Samples of the as-milled and the annealed powders, each about $50 \mathrm{~g}$, were placed in thin-walled aluminum cans and mounted at room temperature on the goniometer of the HB2 


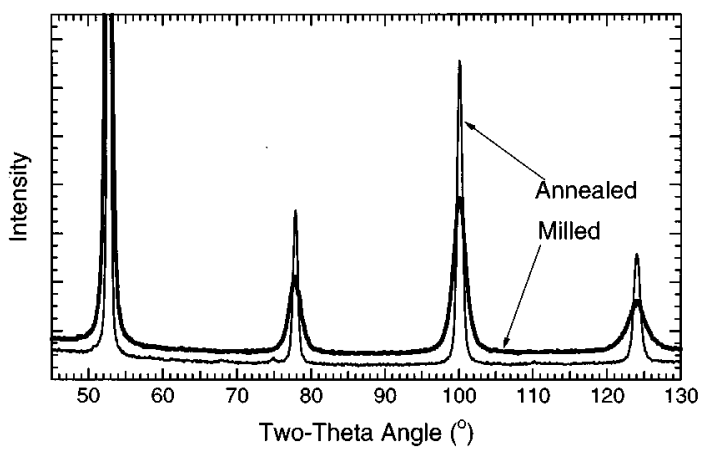

FIG. 1. X-ray powder diffraction patterns of the as-milled (nanocrystalline) and annealed (larger-grained) Fe powders. The as-milled data are offset vertically for clarity.

triple axis spectrometer at the High Flux Isotope Reactor at the Oak Ridge National Laboratory. The spectrometer was operated in constant- $Q$ mode with the fixed final energy $E_{f}$ of $14.8 \mathrm{meV}$. The energy loss spectra were made by scanning the incident energy from 14.8 to $74.8 \mathrm{meV}$. The neutron flux from the monochromator was monitored with a fission detector, which was used to control the counting time for each data point. The incident beam on the pyrolytic graphite monochromator crystal had a collimation of $110^{\prime}$, and $40^{\prime}$ Soller slits were used between the monochromator and the sample. Pyrolytic graphite filters placed after the sample were used to attenuate the $\lambda / 2$ and $\lambda / 3$ contamination. The filtered beam passed through $40^{\prime}$ slits before the pyrolytic graphite analyzer crystal. Following the analyzer, $70^{\prime}$ Soller slits were used before the ${ }^{3} \mathrm{He}$ detector. With this arrangement, the energy resolution varied between 0.9 and $2 \mathrm{meV}$, depending on the energy transfer and the slope of the phonon dispersion surface. Spectra from each specimen were obtained at two values of $Q, 3.98$ and $4.60 \AA^{-1}$.

\section{RESULTS}

Figure 1 presents $x$-ray-diffraction patterns from the asmilled powder and from the same powder after annealing. Grain sizes and distributions of internal strains were obtained by the method of Williamson and Hall, ${ }^{14}$ after correcting the peak shapes for the characteristic broadening of the $\mathrm{x}$-ray diffractometer. In this method the widths of the diffraction peaks in $k$ space were plotted against the $k$ vectors of the diffraction peaks. The slopes of these plots then provide the mean-squared strain, and the $y$ intercepts provide the characteristic crystallite size. We also used the Scherrer method to determine a crystallite size. ${ }^{14}$ Mean crystallite sizes were $12 \mathrm{~nm}$ for the as-milled Fe powder and $28 \mathrm{~nm}$ for the annealed powder. The mean squared strains were $0.4 \%$ in the as-milled, and $0.1 \%$ in the annealed powders. Gas chromatography showed that the as-milled and annealed samples of Fe powder had, respectively, hydrogen contents of $<0.004$ and $0.006 \mathrm{wt} \%$, oxygen contents of 0.236 and $0.262 \mathrm{wt} \%$, and nitrogen contents of 0.748 and 0.849 wt $\%$. Some of these gases, especially oxygen and nitrogen, may have been adsorbed on the surfaces of the powders and not absorbed interstitially. The as-milled and annealed samples of $\mathrm{Ni}_{3} \mathrm{Al}$

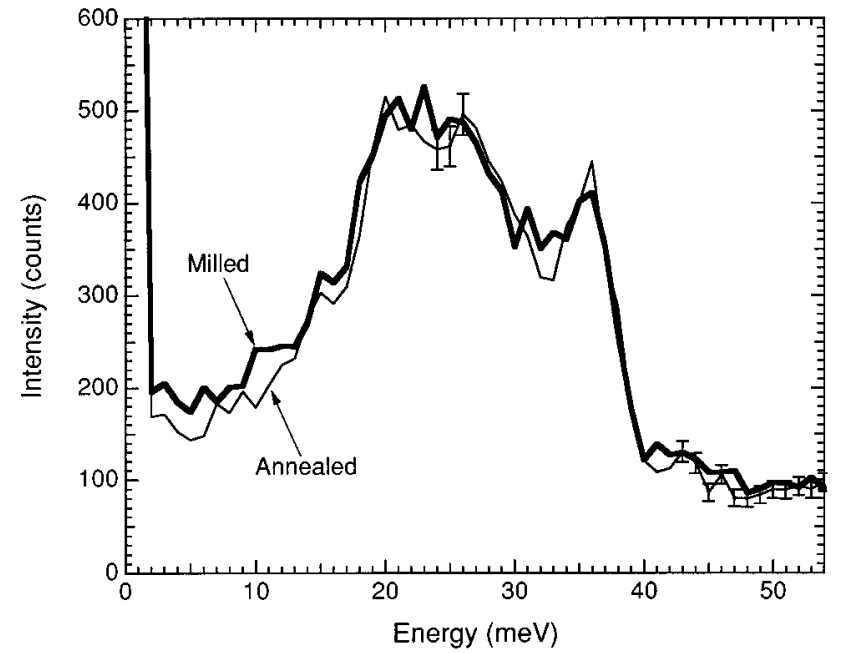

FIG. 2. Sum of neutron energy loss spectra at $Q=3.98$ and $4.60 \AA^{-1}$ for the nanocrystalline and larger-grained powders.

had, respectively, hydrogen contents of 0.0083 and 0.0128 wt $\%$, oxygen contents of 0.0695 and 0.0502 wt $\%$, and nitrogen contents of 0.232 and 0.188 wt $\%$. We do not expect any significant quasielastic neutron scattering from these trace amounts of hydrogen.

The individual energy loss spectra from the Fe powders show a modest dependence on the momentum transfer $Q$, as is generally expected for coherent inelastic scattering from polycrystalline alloys. ${ }^{15}$ Figure 2 presents the sum of the spectra for both values of $Q, 3.98$ and $4.60 \AA^{-1}$. In these summed data, as in the individual pairs of spectra, two differences are seen. First, in the low-energy regions below 15 $\mathrm{meV}$ there is stronger scattering from the as-milled Fe than the annealed Fe. The second difference is that the spectra from the annealed powder have sharper longitudinal peaks (near $36 \mathrm{meV}$ ) than do the spectra from the as-milled powder. This is seen most prominently for the peak of the longitudinal branch at an energy of about $36 \mathrm{meV}$.

\section{ANALYSIS OF PHONON DOS}

The analysis of the scattering data was helped considerably by the availability of interatomic force constants obtained from previous work with single crystals of bcc Fe. Using the force constants of bulk Fe from Ref. 16, the dynamical matrix of the Born-von Kármán model, $\mathbf{D}(\mathbf{k}),{ }^{17}$ was diagonalized for approximately $10^{5}$ values of $\mathbf{k}$ distributed uniformly over the first Brillouin zone. Histogram binning of the resulting eigenfrequencies provided the calculated phonon DOS. For comparison with our experimental data, this calculated phonon DOS was convoluted with a Gaussian function of full width at half-maximum of $3.9 \mathrm{meV}$.

The experimental energy loss spectra include coherent and incoherent scattering. The incoherent scattering has the shape of the phonon DOS weighted by a thermal factor, but the coherent scattering contribution has a shape that is sensitive to the momentum transfer $Q$. In particular, the coherent inelastic scattering at low energies is expected to be relatively weak for our choices of $Q$. This is because the sum 


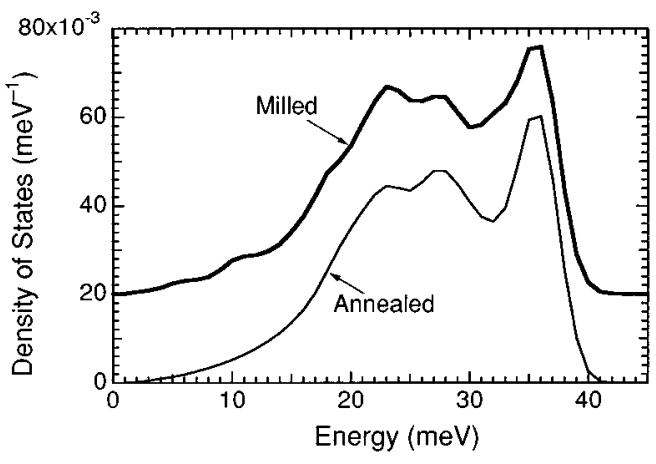

FIG. 3. Phonon DOS curves obtained from the experimental data of Fig. 2, using the calculated phonon DOS to generate a correction factor for converting the annealed Fe spectrum into the Fe phonon DOS. The "instrumentally broadened" phonon DOS from Fe, equal to the corrected phonon DOS of the annealed specimen, is shown as "Annealed." The data from the as-milled sample are offset vertically by 0.02 .

over $\mathbf{q}$ for the dynamical structure factor includes a factor of $\delta(\mathbf{Q}-\mathbf{q}-\tau)$, where $\tau$ is a reciprocal lattice vector, $\mathbf{q}$ is the phonon wave vector, and $\mathbf{Q}$ is the momentum transfer. To obtain the strongest coherent inelastic scattering from phonons with small $q$, the value of $Q$ should be nearly a reciprocal lattice vector. This is not the case for our choices of 3.98 and $4.60 \AA^{-1}$. We devised a correction procedure, described below, to convert the neutron energy loss spectrum of the nanocrystalline powder to a phonon DOS, using the calculated and experimental results from the larger-grained $\mathrm{Fe}$ as a reference.

The features of the inelastic scattering described in the previous section led us to the following procedure for obtaining an approximate phonon DOS from the experimental data. Our data analysis procedure has some similarities to methods used previously. ${ }^{13,18-22}$ The individual spectra for both $Q=3.98$ and $4.60 \AA^{-1}$ were first summed (see Fig. 2). The same constant background was then subtracted from the summed spectra-a constant background is a good approximation for data from the HB2 spectrometer. To understand the thermal factor, the incoherent inelastic scattering was calculated for bcc Fe using the conventional multiphonon expansion. ${ }^{18,20,23}$ We found that at the relatively low values of $Q$ and temperature of the present experiments, multiphonon corrections would have made little difference to the resultant phonon DOS. We therefore divided each background-corrected spectrum by the one-phonon correction factor $(n(\nu)-1) / \nu,{ }^{24}$

$$
\frac{n(\nu)-1}{\nu}=\frac{1}{\nu[1-\exp (-h \nu / k T)]} .
$$

To accommodate the $Q$ dependence of the coherent inelastic scattering, we compared these intermediate results to the calculated phonon DOS of Fe. We obtained a correction function by dividing this calculated DOS by the intermediate result from the annealed Fe powder. When the intermediate result for the annealed bcc Fe was multiplied by this correction function, the calculated curve was of course recovered, and this curve is shown at the bottom of Fig. 3. On the other hand, when the intermediate result from the nanocrystalline,

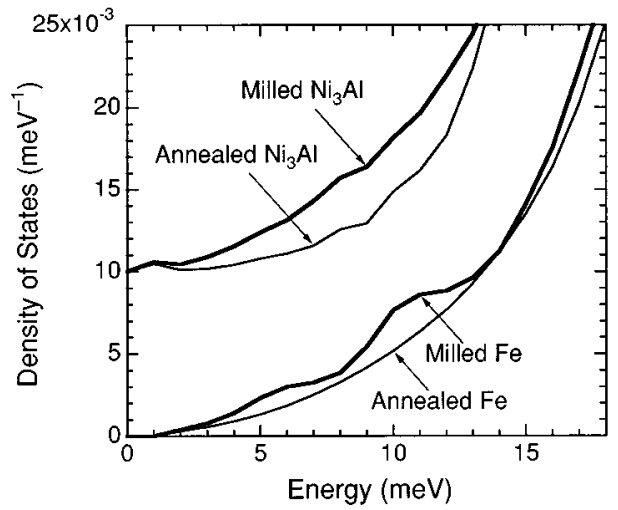

FIG. 4. Enlargement of the data of Fig. 3, together with an enlargement of the phonon DOS for $\mathrm{Ni}_{3} \mathrm{Al}$, published previously (enlarged data from Fig. 4 of Ref. 13).

as-milled Fe was multiplied by this correction function, we obtained the phonon DOS for the nanocrystalline Fe at the top of Fig. 3. Finally, we normalized the areas of the phonon DOS curves. (The normalization correction was small, about $3 \%$, and likely caused by differences in the powder packing in the two sample cans.)

\section{DISCUSSION}

\section{A. High-energy regime}

Figure 3 shows that the "longitudinal peak' at $36 \mathrm{meV}$ is broadened in the as-milled material, in comparison to the peak in the annealed material. Much of the broadening may have been caused by short phonon lifetimes in nanophase material-a confinement effect. A phonon wave packet is expected to travel a distance, $l \sim 12 \mathrm{~nm}$, characteristic of the crystallite size, before scattering. The characteristic number of cycles for the phonon lifetime $y$ is

$$
y=\frac{\nu l}{v},
$$

where $v$ is the sound velocity and $\nu$ is the phonon frequency. For the longitudinal peaks in Fe, Eq. (2) gives $y \sim 20$, suggesting a lifetime broadening of 5\%-10\% in energy. This is a significant effect that can account for the observed broadening. The lifetime broadening should be approximately symmetrical about the mean energy, however, and the longitudinal peak in the experimental spectra is broadened more asymmetrically than expected for a damped harmonic oscillator. There is a distinct shift of some longitudinal frequencies toward lower energies in the nanophase Fe. A lower effective stiffness, presumably caused by crystalline defects, could be responsible for this shift.

\section{B. Low-energy regime}

The second feature of the phonon DOS of the nanophase material that differs observably from that of the annealed $\mathrm{Fe}$ is an enhancement of vibrational modes at low energies. The phonon DOS at low energies is presented in Fig. 4, together with an enlargement of previously published results from as-milled and annealed powders of $\mathrm{Ni}_{3} \mathrm{Al}^{13}$ The effects are 
qualitatively similar, but larger for $\mathrm{Ni}_{3} \mathrm{Al}$ than for $\mathrm{Fe}$. Although we suggested previously that this enhancement of the $\mathrm{Ni}_{3} \mathrm{Al}$ phonon DOS in the low-energy regime could have been caused by quasielastic scattering from hydrogen,${ }^{13}$ the present measurements of hydrogen in the $\mathrm{Ni}_{3} \mathrm{Al}$ powders showed that the as-milled powder actually has less hydrogen than the annealed powder. Recent measurements of the phonon DOS of nanophase and large-grained $\mathrm{Ni}$ by Trampenau et al. ${ }^{25}$ gave results similar those in Fig. 4 for $\mathrm{Ni}_{3} \mathrm{Al}$.

Fecht $^{26}$ and Wagner ${ }^{27}$ proposed a two-phase model for atomic vibrations in nanocrystalline materials, in which the "grain-boundary phase" has vibrations of lower frequency than the "crystalline phase." Perhaps some of the softening of the high-frequency modes described in Sec. V A can be understood as atom vibrations within the grain-boundary region. It is harder to understand our observed enhancements in the low-energy regime with a two-phase model, however. It is unlikely that long-wavelength phonons can exist within a grain boundary. It is also unlikely that the shortwavelength phonons within a grain boundary can be suppressed in energy from $30 \mathrm{~V}$ to around $3 \mathrm{meV}$, since this implies a softening of the effective interatomic force constants by a factor of 100 .

We suggest that some enhancement of the phonon DOS at low energies could originate from intercrystalline vibrations of the nanocrystalline microstructure. It is important to ask what condition is required for the low-energy DOS of a nanocrystalline microstructure to be comparable to or larger than the phonon DOS of a crystal of individual atoms. In the Debye model, or for phonons at low $q$ characterized by a constant velocity of sound, we have a DOS $g(\nu)$

$$
g(\nu)=\frac{9 N}{\nu_{D}^{3}} \nu^{2} .
$$

The Debye frequency is

$$
\nu_{D} \propto \sqrt{k / m},
$$

where $k$ is an averaged inter-atomic force constant and $m$ is an atomic mass. In lumping $n$ atomic masses into a nanocrystal, we have a new DOS for the nanocrystalline microstructure $g^{\prime}(\nu)$,

$$
g^{\prime}(\nu)=\frac{9 N}{n \nu_{D}^{\prime 3}} \nu^{2},
$$

where the Debye frequency for the nanocrystalline microstructure $\nu_{D}^{\prime}$ is

$$
\nu_{D}^{\prime} \propto \sqrt{k^{\prime} / n m},
$$

and $k^{\prime}$ is an average intercrystallite force constant. To obtain a high density of states in the nanocrystalline microstructure, we seek the condition $g^{\prime}(\nu)>g(\nu)$. We formulate this condition with Eqs. (3),(4) and (5),(6),

$$
\begin{aligned}
& \frac{9 N}{n}\left(\frac{n m}{k^{\prime}}\right)^{3 / 2} \nu^{2}>9 N\left(\frac{m}{k}\right)^{3 / 2} \nu^{2}, \\
& n^{1 / 3} k>k^{\prime} .
\end{aligned}
$$

For a nanocrystalline microstructure to have an enhancement in its DOS at low energies that is comparable to the
DOS itself, Eq. (8) requires that the intercrystallite force constant must increase more slowly than the linear dimension of the crystallite $l$. For a two-dimensional grain-boundary between crystallites, whose area scales as $l^{2}$, this demands that the average interatomic bond across the grain-boundary decreases in strength inversely with $l$. The stiffness of grain boundaries in nanocrystalline materials is largely unknown, but we do expect that the interatomic force constants across a grain boundary are weaker than in regions of good crystalline material. The intercrystallite force constants are probably sensitive to the particular material and its processing. It therefore seems plausible that there could be a significant difference in the intercrystallite force constants of our nanocrystalline $\mathrm{Fe}$ and nanocrystalline $\mathrm{Ni}_{3} \mathrm{Al}$. Since the nanocrystalline $\mathrm{Ni}_{3} \mathrm{Al}$ had a crystallite size of $7 \mathrm{~nm}$, versus 10 and $12 \mathrm{~nm}$ for the nanocrystalline Ta (Ref. 12) and Fe, it is also possible that the requirement of Eq. (8) may be better satisfied by nanocrystalline $\mathrm{Ni}_{3} \mathrm{Al}$ than nanocrystalline $\mathrm{Fe}$ or $\mathrm{Ta}$, giving a larger enhancement of the phonon DOS of $\mathrm{Ni}_{3} \mathrm{Al}$ at low energies. In addition to our uncertainty of the intercrystallite force constants, however, we have important uncertainties about the lattice dynamics of an irregular nanocrystalline microstructure, such as the coupling between inter- and intracrystallite vibrations.

\section{Vibrational entropy}

We define $\Delta S_{\text {vib }} \equiv S_{\text {vib }}^{\text {nan }}-S_{\text {vib }}^{\mathrm{gg}}$ as the difference in vibrational entropy of two states of a material, nanocrystalline and larger grained, at high temperatures. This difference in vibrational entropy depends in a straightforward way on the difference in the phonon DOS of the two phases, $g^{\operatorname{nan}}(\nu)-g^{\lg }(\nu),{ }^{13}$

$$
\Delta S_{\text {vib }}=-3 k_{B} \int_{0}^{\infty}\left[g^{\operatorname{nan}}(\nu)-g^{\lg }(\nu)\right] \ln (\nu) d \nu,
$$

where the difference avoids problems with the dimensions of the argument of the logarithm. Using the phonon DOS shown in Fig. 3, we find $\Delta S_{\text {vib }}=0.019 k_{B} /$ atom for the difference in vibrational entropy between the as-milled nanophase $\mathrm{Fe}$ and the annealed larger-grained $\mathrm{Fe}$. This difference in vibrational entropy contributes only a small amount to the thermodynamic stability of the nanophase microstructure, giving a $-T\left(\Delta S_{\text {vib }}\right)$ term in the free energy of $-48 \mathrm{~J} / \mathrm{mol}$ at $300 \mathrm{~K}$. In comparison, the excess enthalpy of nanophase materials is a few $\mathrm{kJ} / \mathrm{mol}^{28}$ Although we do not find a large entropy for nanocrystalline $\mathrm{Fe}$ as suggested previously, ${ }^{26,27}$ it is possible that $\Delta S_{\text {vib }}$ may be larger for nanocrystalline materials having weaker intercrystallite force constants and crystallite sizes smaller than $12 \mathrm{~nm}$.

\section{CONCLUSION}

We performed inelastic neutron scattering experiments on Fe powders in two microstructural states: as nanocrystals prepared by high-energy ball milling and as larger crystallites prepared by annealing. For each sample, neutron energy loss spectra were collected at two values of $Q$. A Born-von Kármán analysis was performed with the force constants of bcc Fe and the results from this analysis provided a correc- 
tion factor for converting our experimental results from the larger-grained, annealed material to a phonon DOS of bcc Fe. The spectra from the as-milled nanocrystalline powder were also analyzed with this method, so that differences in the phonon DOS could be identified.

We found two differences in the phonon DOS of the nanocrystalline and larger-grained $\mathrm{Fe}$ powders. At highenergy we found a broadening of the longitudinal peak in the nanocrystalline powder. This could be caused primarily by lifetime broadening. It also seems that in the nanocrystalline material the longitudinal modes are shifted downward in energy. A second difference in the phonon DOS occurred in the low-energy regime below about $15 \mathrm{meV}$, where the phonon DOS of the nanocrystalline material was enhanced over that of the larger-grained material. We attribute this enhancement of the phonon DOS to intercrystallite vibrational modes of the nanocrystalline microstructure. These measured changes in phonon DOS should have only a small effect on the vibrational entropy of nanocrystalline Fe prepared by highenergy ball milling.

\section{ACKNOWLEDGMENTS}

The Oak Ridge National Laboratory is managed for the Department of Energy by Lockheed Martin Energy Research, Oak Ridge, TN, under Contract No. DE-AC0596OR22464. This work was supported by the U.S. Department of Energy under Contract No. DE-FG-03-86ER45270.

${ }^{1}$ S. Schuppler, S. L. Friedman, M. A. Marcus, D. L. Adler, Y. H. Yie, F. M. Ross, T. D. Harris, W. L. Brown, Y. J. Chabal, et al., Phys. Rev. Lett. 72, 2648 (1994).

${ }^{2}$ H. Karch, R. Birringer, and H. Gleiter, Nature 330, 556 (1987).

${ }^{3}$ X. Zhu, R. Birringer, U. Herr, and H. Gleiter, Phys. Rev. B 35, 9085 (1987); T. Haubold, R. Birringer, B. Lengeler, and H. Gleiter, Phys. Lett. A 135, 461 (1989).
${ }^{4}$ M. R. Fitzsimmons, J. A. Eastman, M. Müller-Stach, and G. Wallner, Phys. Rev. B 44, 2452 (1991).

${ }^{5}$ B. Fultz, H. Kuwano, and H. Ouyang, J. Appl. Phys. 76, 5961 (1994).

${ }^{6}$ H. Gleiter, Prog. Mater. Sci. 33, 223 (1989).

${ }^{7}$ A. Tschöpe and R. Birringer, Acta Metall. Mater. 41, 2791 (1993).

${ }^{8}$ G. Von Eynatten, J. Horst, K. Dransfeld, and H. E. Bömmel, Hyperfine Interactions 29, 1311 (1986).

${ }^{9}$ J. A. Eastman and M. R. Fitzsimmons, J. Appl. Phys. 77, 522 (1995).

${ }^{10}$ M. Hayashi, E. Gerkema, A. M. van der Kraan, and I. Tamura, Phys. Rev. B 42, 9771 (1990).

${ }^{11}$ L. B. Hong, C. C. Ahn, and B. Fultz, J. Mater. Res. 10, 2408 (1995).

${ }^{12}$ K. Suzuki and K. Sumiyama, Mater. Trans. JIM 36, 188 (1995).

${ }^{13}$ B. Fultz, L. Anthony, L. J. Nagel, R. M. Nicklow, and S. Spooner, Phys. Rev. B 52, 3315 (1995).

${ }^{14}$ H. P. Klug and L. E. Alexander, X-Ray Diffraction Procedures (WileyInterscience, New York, 1974), pp. 656 and 664.

${ }^{15}$ F. W. de Wette and A. Rahman, Phys. Rev. 176, 784 (1968).

${ }^{16}$ V. J. Minkiewicz, G. Shirane, and R. Nathans, Phys. Rev. 162, 528 (1967).

${ }^{17}$ G. Venkataraman, L. A. Feldkamp, and V. C. Sahni, Dynamics of Perfect Crystals (MIT Press, Cambridge, 1975), Chap. 2.

${ }^{18}$ J.-B. Suck and H. Rudin, in Glassy Metals II, edited by H. Beck and H.-J. Güntherodt (Springer, Berlin, 1983), Chap. 7.

${ }^{19}$ J. Chevrier, J.-B. Suck, M. Perroux, and J. J. Caponi, Phys. Rev. Lett. 61, 554 (1988).

${ }^{20}$ D. D. Klug, E. Whalley, E. C. Svensson, J. H. Root, and V. F. Sears, Phys. Rev. B 44, 841 (1991).

${ }^{21}$ C.-K. Loong, P. Vashishta, R. K. Kalia, W. Jin, M. H. Degani, D. G. Hinks, D. L. Price, J. D. Jorgensen, B. Dabrowski, A. W. Mitchell, D. R. Richards, and Y. Zheng, Phys. Rev. B 45, 8052 (1992).

${ }^{22}$ J. Chevrier, J.-B. Suck, J. C. Lasjanunias, M. Perroux, and J. J. Caponi, Phys. Rev. B 49, 961 (1994).

${ }^{23}$ V. F. Sears, Phys. Rev. A 7, 340 (1973).

${ }^{24}$ G. Kostorz and S. W. Lovesey, in Treatise on Materials Science and Technology Vol. 15: Neutron Scattering, edited by G. Kostorz (Academic, New York, 1979), p. 1.

${ }^{25}$ J. Trampenau, K. Bauszuz, W. Petry, and U. Herr, Nanostructured Mater. 6, 551 (1995).

${ }^{26}$ H. J. Fecht, Phys. Rev. Lett. 65, 610 (1990).

${ }^{27}$ M. Wagner, Acta Metall. Mater. 40, 957 (1992).

${ }^{28}$ H. J. Fecht, E. Hellstern, Z. Fu, and W. L. Johnson, Adv. Powder Metall. 1-3, 111 (1989). 